\title{
Effect of an Axial Magnetic Field on the Performance of a Quadrupole Mass Spectrometer
}

\author{
Sarfaraz U. Syed, Jeyan Sreekumar, Boris Brkic, John R. Gibson, and \\ Stephen Taylor \\ Department of Electrical Engineering and Electronics, University of Liverpool, Liverpool, United Kingdom
}

\begin{abstract}
We consider the case of a quadrupole mass spectrometer (QMS) in which a static magnetic field is applied axially in the $z$-direction along the length of the mass filter. The theoretical approach assumed in the model is that the QMS contains hyperbolic rods as electrodes and that the magnetic field acts over the full length of the mass filter assembly. Initial experimental results with argon and helium for a low-resolution instrument confirm the predicted theoretical trends. The analysis also predicts for which values of operating parameters an enhancement of the instrument resolution is achieved when an axial magnetic field is applied. The model predicts instrument resolution $\mathrm{R}>3000$ for a QMS with a $200 \mathrm{~mm}$ long mass filter via application of an axial magnetic field. (J Am Soc Mass Spectrom 2010, 21, 2070-2076) (C) 2010 Published by Elsevier Inc. on behalf of American Society for Mass Spectrometry
\end{abstract}

$\mathrm{F}$ irst described by W. Paul and H. Steinwedel in 1953 [1], a quadrupole mass spectrometer (QMS) is used to measure molecular masses and relative composition based on mass-to-charge $\mathrm{m} / \mathrm{z}$ ratio of charged particles. It consists primarily of the following three components: an ion source, a mass analyzer, and an ion detector [2]. Examples of mass analyzers include the quadrupole mass filter (QMF) [3], ion trap [4,5], and time of flight MS [6], each of which provide different performance and operational characteristics. Attributes of the QMS such as versatility, accuracy, low cost, mass range, and sensitivity have ensured that it has been deployed in a wide range of applications, varying considerably from the modest residual gas analyzer to high-performance mass spectrometer for chemical analysis of simple and complex molecules, e.g., when used in conjunction with gas chromatography $[7,8]$. Different applications have different requirements for resolution, sensitivity, and stability.

There have been many analytical predictions of the behavior of QMS. Dawson used matrix methods to calculate ion transmission for various scan lines and various apertures both with and without fringing fields, based on maximum ion displacements for mass filters [9]. Later research describes the performance of the QMS more comprehensively by solving the Mathieu equation in two dimensions for an infinitely long mass filter [2]. Batey showed that some features of the behavior of the QMS could be predicted by tracing the motion of ion through the mass filter [10]. Muntean used the

Address reprint requests to Dr. S. Taylor, Department of Electrical Engineering and Electronics, University of Liverpool, Brownlow Hill, Liverpool L69 3GJ, U.K. E-mail: s.taylor@liv.ac.uk matrix method to develop a computer simulation program to model ion transmission through the filter by calculating ion trajectories in radio frequency (rf) only quadrupoles [11]. More examples of such types of analytical work include the modeling of ion transmission through the filter by calculating ion trajectories in exactly determined hyperbolic quadrupole fields [12], the effects of $\mathrm{rf}$ frequency, phase and magnitude on the performance of QMS [13], and the effects of initial ion energy and quadrupole rod length on transmission percentage of ions through the mass filter along with the effects of aperture parameters [14]. Some workers have developed computational methods to determine the trajectories of large number of ions in QMS; their computer program generates large number of ions (at least $10^{5}$ ions injected into the quadrupole model at each point on the mass scale), thus providing a detailed computer simulation for both hyperbolic and circular rods $[15,16]$. Douglas and Konenkov have used numerical calculations to investigate the influence of electrode radius $r$ to field radius $r_{0}$, which is referred to as $\left(r / r_{0}\right)$ on the peak shape for a linear QMF constructed with round rods [17]. Other workers have used commercially available software tools, such as SIMION 3D, to compute field conditions and ion trajectories of a commercial QMS with a number of imperfections, as compared to ideal hyperbolic geometry [1]. Hogan and Taylor performed computer simulation with a large number of ions $\left(10^{8}\right)$ to investigate the operation of QMS in first and third stability zones, and it was concluded that QMS operation in zone 3 of the Mathieu stability diagram provides an improved immunity from the effects of the variation in the value of $\left(r / r_{0}\right)$ compared to zone 1 [18]. More recently, Sreekumar et al. performed 
simulations with a large number of ions $\left(>10^{7}\right)$ to determine the required resolution for the qualitative and quantitative identification of low mass isotopes in the mass range 1-6 $u$ [19].

The two main operating characteristics of a quadrupole mass filter are the mass range and the maximum resolution. The maximum resolution and mass range are dependent upon six basic parameters; these are the length and the shortest distance between the rods $\left(2 r_{0}\right)$, the maximum DC supply voltage to the rods, the rf frequency of the quadrupole, the rf supply voltage, and the ion injection energy. To improve the maximum resolution, the dimensions of the QMF or the operating parameters are to be changed. However, geometric modifications to improve performance increase the manufacturing cost and usually the size of the instrument. A low cost method to increase the resolution of a given QMS is the application of magnetic field to the mass filter [20,21], and is the motivation behind this study. Magnetic fields have long been used in ion traps, quadrupole ion sources, and magnetic sector instruments; however, little work has been done in relation to the application of magnetic field to the QMS. Tunstall et al. found experimentally that the application of static transverse magnetic field to the mass filter of QMS increases the resolution by reducing the long tail on low mass side of the mass spectra [20]. Later work showed the effect of static magnetic field on ion trajectories and resolution. Simulations showed that an applied transverse magnetic field in the $x$-direction has no effect on ion motion in the $x$-direction; however, ion motion in the $y$-direction is both displaced and increased in amplitude, and that this can result in an improvement in QMS performance [21]. The original model [20,21] was modified for better sensitivity (using a larger number of steps along the mass scale) and to include large number of ions at each point on the mass scale. Some workers have described an rf-only ion trap mode, with high magnetic fields applied to overcome problems such as the low charge state of ions and ion focusing [22]. This method has been used for in-field matrix-assisted laser desorption ionization Fourier transform mass spectrometry. It was found that for a larger ion trap, a high value of applied magnetic field increases the resolution.

In this paper, therefore, we extend the previous work and present new theoretical and experimental results for a QMS operating under a static magnetic field applied in the $z$-direction, i.e., axially along the length of the mass filter. The numerical model used in the simulation assumes that the QMF contains hyperbolic electrodes and that the magnetic field penetrates along the full length of the filter.

\section{Theory}

Ions oscillating tangentially to a magnetic field will experience a Lorentz force $F=q(E+v \times B)$ where $v$ is the instantaneous velocity of the particle and is directly proportional to ion energy $E_{z}, q$ is electric charge of the particle, $E$ is the strength of electric field and $B$ is the strength of magnetic field [23]. If the electric field is given by conventional hyperbolic potential where $U$ is the amplitude of DC potential applied to rods, $V$ the amplitude of rf potential, $f$ the frequency of the sinusoidal field and $r_{0}$ the inscribed radius of the electrodes, for general magnetic field $B=\left(B_{x}, B_{y}, B_{z}\right)$ the coupled equations of motion are given by

$$
\begin{aligned}
& \frac{d^{2} x}{d^{2} \xi}=-x(a-2 q \cos 2 \xi)+\left(\frac{d y}{d \xi} b_{3}-\frac{d z}{d \xi} b_{2}\right) \\
& \frac{d^{2} y}{d^{2} \xi}=y(a-2 q \cos 2 \xi)+\left(\frac{d z}{d \xi} b_{1}-\frac{d x}{d \xi} b_{3}\right) \\
& \frac{d^{2} z}{d^{2} \xi}=\left(\frac{d x}{d \xi} b_{2}-\frac{d y}{d \xi} b_{1}\right)
\end{aligned}
$$

In the numerical model the above equations have been written in a dimensionless form where the only dimension that appears is that of length displacement. The time $t$ has become $\mathrm{t}=2 \xi / \omega$ where $\omega$ is the angular frequency equal to $2 \pi f$. In the absence of an applied magnetic field the direct potential $U$ and alternating potential $V$ are related to $a$ and $q$ as

$$
\begin{aligned}
& a=\left(\frac{4 e U}{m r_{0}^{2} \omega^{2}}\right) \\
& q=\left(\frac{2 e V}{m r_{0}^{2} \omega^{2}}\right)
\end{aligned}
$$

The components of the magnetic field $B$ are

$$
\left(b_{1}, b_{2}, b_{3}\right)=\left(\frac{2 e B_{x}}{m \omega}, \frac{2 e B_{y}}{m \omega}, \frac{2 e B_{z}}{m \omega}\right)
$$

It can be seen from eqs 1-3 that if the magnetic field is taken to be zero the coupled differential equations reduce to the familiar Mathieu equations for a linear quadrupole. Solving numerically the coupled differential equations gives the trajectories of the ions through the mass filter. From the trajectories it is feasible to determine the conditions which give successful ion transmission for a given mass to charge ratio. This depends on the values of direct potential $U$, the $\mathrm{rf}$ potential $V$, the $\mathrm{rf}$ voltage frequency $f$, the inscribed radius of the quadrupole rods $r_{0}$ the magnetic field strength $B$, the initial velocity, initial phase and the ion position. The first four parameters appear as the variables of $a$ and $q \ell$ in the Mathieu equation for $(B=0)$. For $B$ non-zero there will be a further dependence of $a$ and $q$ upon the magnetic field, however a complete theoretical analysis is beyond the scope of this paper. For simplicity we have assumed that the stability diagram for non-zero $B$ is similar in shape to the case for 
$B=0$ and can be approximated by the stability diagram for $B=0$. From the mass spectrum obtained by the numerical simulation, the resolution $R$ can be found by using the equation $R=M / \Delta M$, where $M$ is the mass of the given spectral peak and $\Delta M$ is the width of the mass peak measured at $10 \%$ of its height [2].

\section{Simulation Method}

A custom software program (QMS-hyperbolic) is used in this study. The program calculates ion trajectories by solving the Mathieu equations using a fourth order Runge-Kutta algorithm. It operates by dividing the ion trajectories into small steps and assuming that over the steps the ion motion in three directions $x, y$, and $z$ may be uncoupled. Mass scans are computed by ramping the values of $U$ and $V$ with fixed $U / V$ ratio, which sets the resolution of an instrument. An option of introducing magnetic field in all the three directions $\left(B x, B_{y}, B_{z}\right)$, along the whole length of the mass filter is also incorporated.

A second program (IonSrc) allows entry conditions for large number of ions (typically $10^{6}$ ), to be specified, which are subsequently supplied to the mass filter calculation engine to simulate individual trajectories in each case. The IonSrc file assumes a uniformly illuminated ion distribution across a user defined ion source exit radius. Each ion is injected into the QMF with random phase with respect to the rf at the time of entry. Finally, Matlab and Microsoft Excel were used to post process the data and for the generation of graphical results.

\section{Experimental Results}

Reported here are the experimental mass peaks for ${ }^{40} \mathrm{Ar}^{+}$and ${ }^{4} \mathrm{He}^{+}$ions without and with an applied magnetic in the $z$-direction. A conventional single filter QMS instrument supplied by MKS Spectra Products (Crewe, UK) was used. The QMF length was $100 \mathrm{~mm}$ with an electrode diameter of $6.35 \mathrm{~mm}$ and field radius of $2.76 \mathrm{~mm}$, excited at $1.8342 \mathrm{MHz}$. The instrument was operated in Faraday only mode and in this configuration typically used as a residual gas analyzer (RGA). The QMS was housed in a stainless steel chamber pumped by a turbo pump, backed by a rotary backing pump providing with a residual gas pressure of $2 \times$ $10^{-6}$ Torr. After admitting the sample gas into the vacuum chamber the working pressure was raised to about $1 \times 10^{-4}$ Torr. An axial magnetic field is applied in the $z$-direction of the mass filter by passing a direct current through a solenoid coil wrapped on the stainless steel chamber housing the QMS exterior to the electrode length. The magnetic field is generated by applying a fixed value of current from a DC supply and measured using Hall effect sensor. The value of magnetic flux density was adjusted at $0.015 \mathrm{~T}$ for experiments with Argon, whereas for the case with helium it was set at $0.007 \mathrm{~T}$. The resolution was adjusted by setting ion energy to a value of $4.5 \mathrm{eV}$, this also gives an easily measurable ion current. The emission current was adjusted as $0.5 \mathrm{~mA}$ and the electron energy in the QMS ion source was set to $70 \mathrm{eV}$. The magnetic field extended $80 \%$ of the length of QMF.

The QMS used the Microvision Plus software (MKS Spectra Products, Crewe, UK) with a fully automated high stability data acquisition electronic drive unit for fast dynamic scanning of the mass filter electrode voltages. The start mass, end mass (range), number of steps, sweep rate, and averaging were user selected. A visual display of each mass scan was displayed in real time on the PC.

To demonstrate the effect of magnetic field in the $z$-direction on resolution of the QMF, ${ }^{40} \mathrm{Ar}^{+}$and ${ }^{4} \mathrm{He}^{+}$ mass peaks was obtained with and without the magnetic field $B_{z}$ applied. Figure 1a shows typical mass peaks obtained experimentally from the QMS for ${ }^{40} \mathrm{Ar}^{+}$, whereas Figure $1 \mathrm{~b}$ shows mass peaks obtained experimentally for ${ }^{4} \mathrm{He}^{+}$. From both figures it can be seen that there is a clear improvement in resolution at $10 \%$ peak height with magnetic field applied, from approximately $\mathrm{R}=13$ to $\mathrm{R}=19$ for the case with argon and from $\mathrm{R}=4.1$ to $\mathrm{R}=6.1$ for the case with helium. Although such instrument resolutions are low in comparison with more
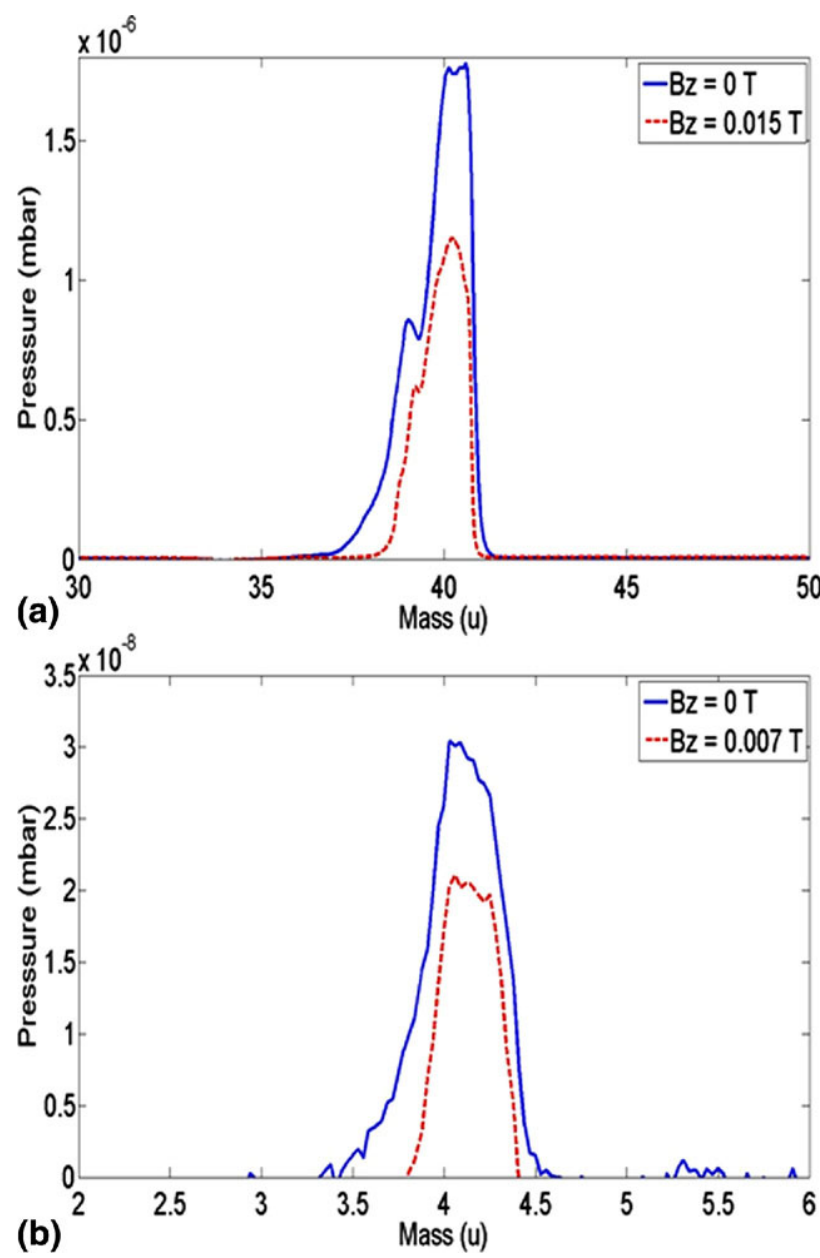

Figure 1. (a) Experimental mass peaks for ${ }^{40} \mathrm{Ar}^{+}$with and without magnetic field applied $(\mathrm{Bz}=0.015 \mathrm{~T})$; $(\mathbf{b})$ experimental mass peaks for ${ }^{4} \mathrm{He}^{+}$with and without magnetic field applied $(\mathrm{Bz}=0.007 \mathrm{~T})$. 
sophisticated instruments (e.g., triple filter QMFs), this experimental result clearly illustrates the resolution enhancement provided by an axially applied magnetic field.

\section{Simulation Results}

\section{The Effect of Magnetic Field in the z-Direction on Ion Trajectories}

The simulation program includes a trace facility, and this allows many details of individual ions to be recorded. Figure 2 shows typical behavior of the ion trajectories calculated using the theoretical model for ions transmitted with and without the axial magnetic field applied. All the simulations were carried out in stability zone 1 (i.e., $a=0.23,699$ and $q=0.706$ ). The corresponding traces shown in the figure are for the same ion injected into the mass filter at the same point in time ( $r f$ phase) and space and at the same point on the mass scale $(m=39.96 \mathrm{u})$. The upper trace of the figure is for the case with no field applied and lower trace with $B_{z}$ of $0.03 \mathrm{~T}$. Ion motion in Figure 2 originates at $x=2.45 \times 10^{-4} \mathrm{~m}$ and $y=7.70 \times 10^{-6} \mathrm{~m}$ with initial velocity of $4.90 \times 10^{3} \mathrm{~m} / \mathrm{s}$. In the lower trace, due to the Lorentz force provided by $B_{z}$, the amplitude of ion trajectory along $y$-direction increases and the amplitude of the trajectory oscillations in the $x$-direction decreases. The application of magnetic field therefore displaces the ion in the $y$ direction so that the ion is lost through impact with the electrodes. It should be noted that ion motion in both the $x$ - and $y$-directions is affected if the magnetic field is applied in the $z$-direction. This is in contrast to the case of magnetic field application in the $x$-direction, where ion motion only in $y$-direction is affected [20].

The Effect of Magnetic Field in z-Direction on the Resolution of QMS

The simulated mass peaks for ${ }^{40} \mathrm{Ar}^{+}$ions using the computer software are shown in Figure 3. All the simulations used 200 steps across the mass range, with $1.5 \times 10^{5}$ ion trajectories run at each point on the peak. Mass peaks for ${ }^{40} \mathrm{Ar}^{+}$are generated for a hyperbolic quadrupole mass filter with length $(l)$ of $100 \mathrm{~mm}$. The inscribed radius of the QMF was taken to be $1.5 \mathrm{~mm}$. The frequency of the rf voltage used in the simulation was $5 \mathrm{MHz}$ and the ion energy was chosen as $5 \mathrm{eV}$. The ion source radius $\left(r_{i e}\right)$ was selected as $0.5 \mathrm{~mm}$ and the exit radius was chosen as $3 \mathrm{~mm}$. The operating point $(U / V$ ratio) was selected as $99.99 \%$, where $100 \%$ corresponds to the intersection of the scan line with the peak of the Mathieu stability diagram at $U / V=0.168$. Figure 3a shows the behavior of the resolution, measured at $10 \%$ peak height as a function of axial magnetic field for three different lengths of QMF. For $\mathrm{L}=100 \mathrm{~mm}$ the resolution

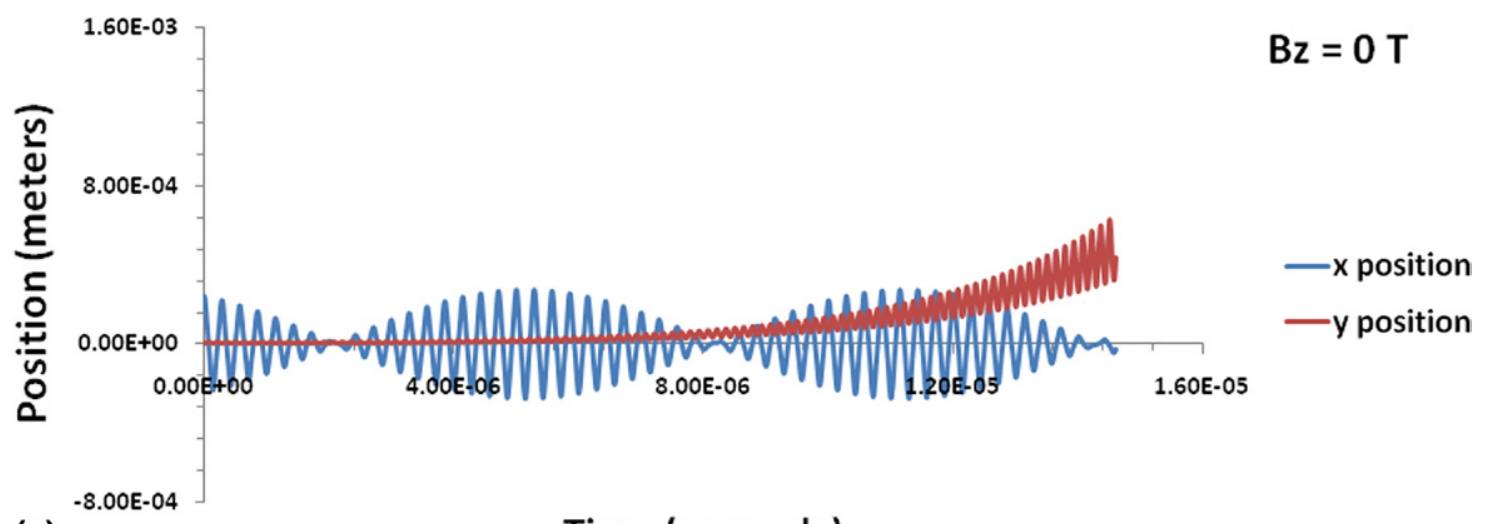

(a)
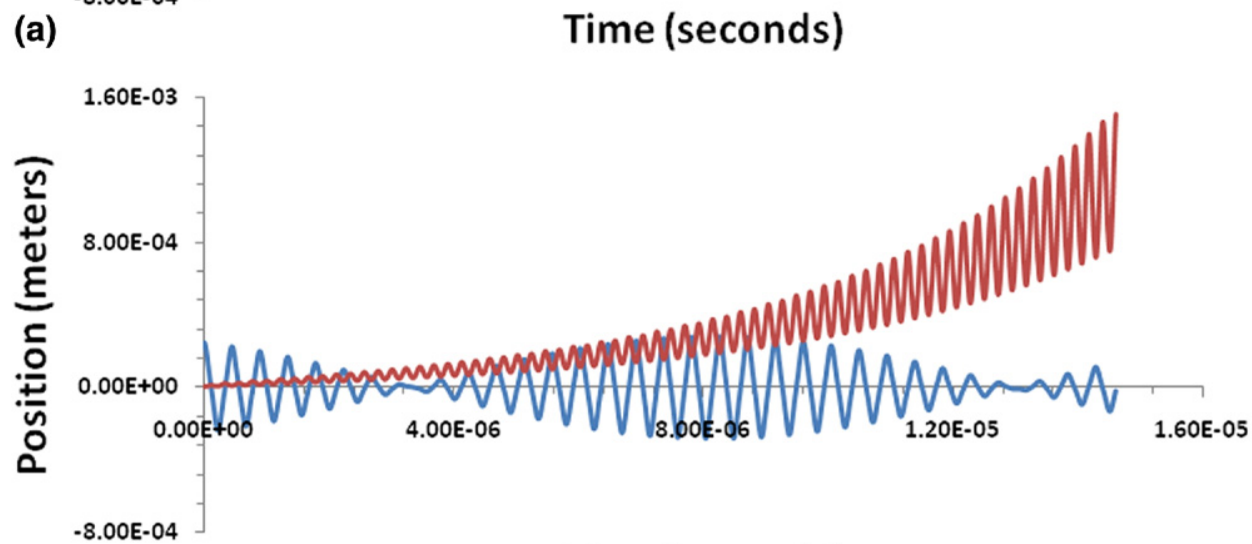

$\mathrm{Bz}=0.03 \mathrm{~T}$

(b)

\section{Time (seconds)}

Figure 2. Numerical simulation of ion trajectories in $\mathrm{x}$ and $\mathrm{y}$ directions for ${ }^{40} \mathrm{Ar}{ }^{+}$with (a) $\mathrm{Bz}=0$ (upper trace) and (b) $\mathrm{Bz}=0.03 \mathrm{~T}$ (lower trace). 

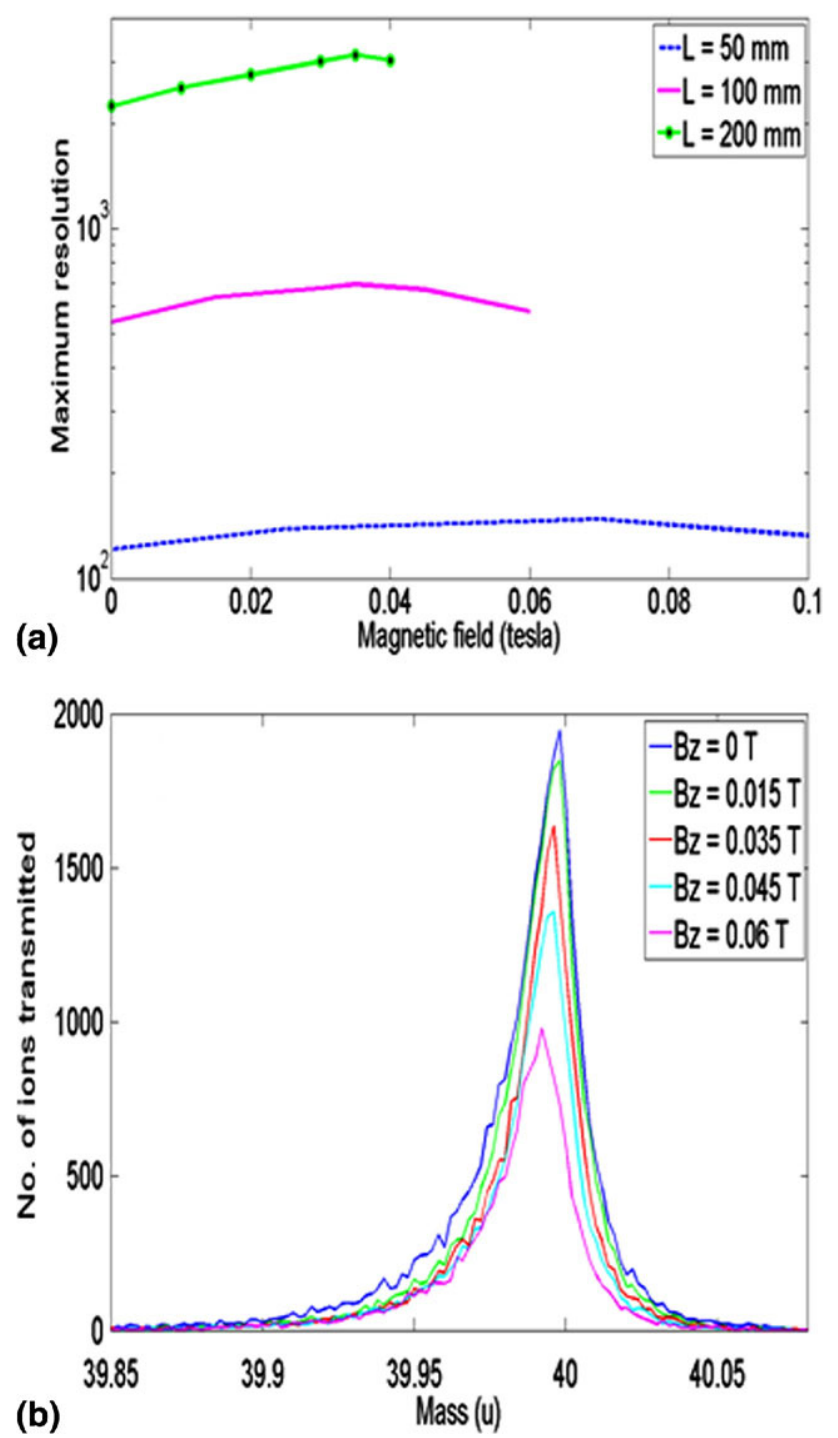

Figure 3. (a) The effect of magnetic field on resolution applied in z-direction (solid line $\mathrm{L}=100 \mathrm{~mm}$, dotted $\mathrm{L}=50 \mathrm{~mm}$, hyphenated $\mathrm{L}=200$ ), (b) simulated mass peaks for ${ }^{40} \mathrm{Ar}^{+}$with and without magnetic field for $\mathrm{L}=100 \mathrm{~mm}$.

increases as $B z$ is varied between 0 and $0.035 \mathrm{~T}$, and then decreases as it is varied between 0.035 and $0.06 \mathrm{~T}$, indicating that the QMS resolution will not increase indefinitely with $B z$. This is because as $B z$ increases, the peak shape eventually degrades. A similar effect is seen for shorter and longer QMF: resolution rises with applied $B z$ reaching a maximum value, then decreases. In the case of $L=200$ $\mathrm{mm}$, the predicted value of resolution is in excess of 3000 which, if achieved in practice, would allow specialist high-resolution RGA applications.

Figure $3 \mathrm{~b}$ shows a set of peaks for ${ }^{40} \mathrm{Ar}^{+}$within a hyperbolic QMF at different values of magnetic field $(B z)$ for a QMF of length $\mathrm{L}=100 \mathrm{~mm}$. An increase in resolution is clearly observed up to a magnetic field of 0.035T. Transmission through the QMF decreases as magnetic field increases, which results in reduction of QMS resolution at higher values of magnetic field 0.045 to 0.06
T. A low-amplitude structure is present across the low mass and high mass sides of the mass peaks, due to minor changes in the acceptance of the QMF as the position of the mass scan changes. This is because the initial phase space positions of a large number of ions are very close to the QMF acceptance boundary, resulting in small local variations of transmission as the mass scan traverses the mass peak. This observed structure on the peak is an artifact of the simulation and can be reduced by increasing the number of ions although at the expense of increased simulation time [17].

The Performance of the QMS and the Effect of Ion Energy on Resolution in the Presence of Magnetic Field in the z-Direction

Figure 4a shows the dependence of maximum resolution on number of rf cycles without and with an applied magnetic field. The relationship between maximum resolution and number of $\mathrm{rf}$ cycles $(N)$ is well known and is given by $M / \Delta M=N^{n} / K$, where $n$ is the slope and $K$ is a constant [2]. For simulations without the magnetic
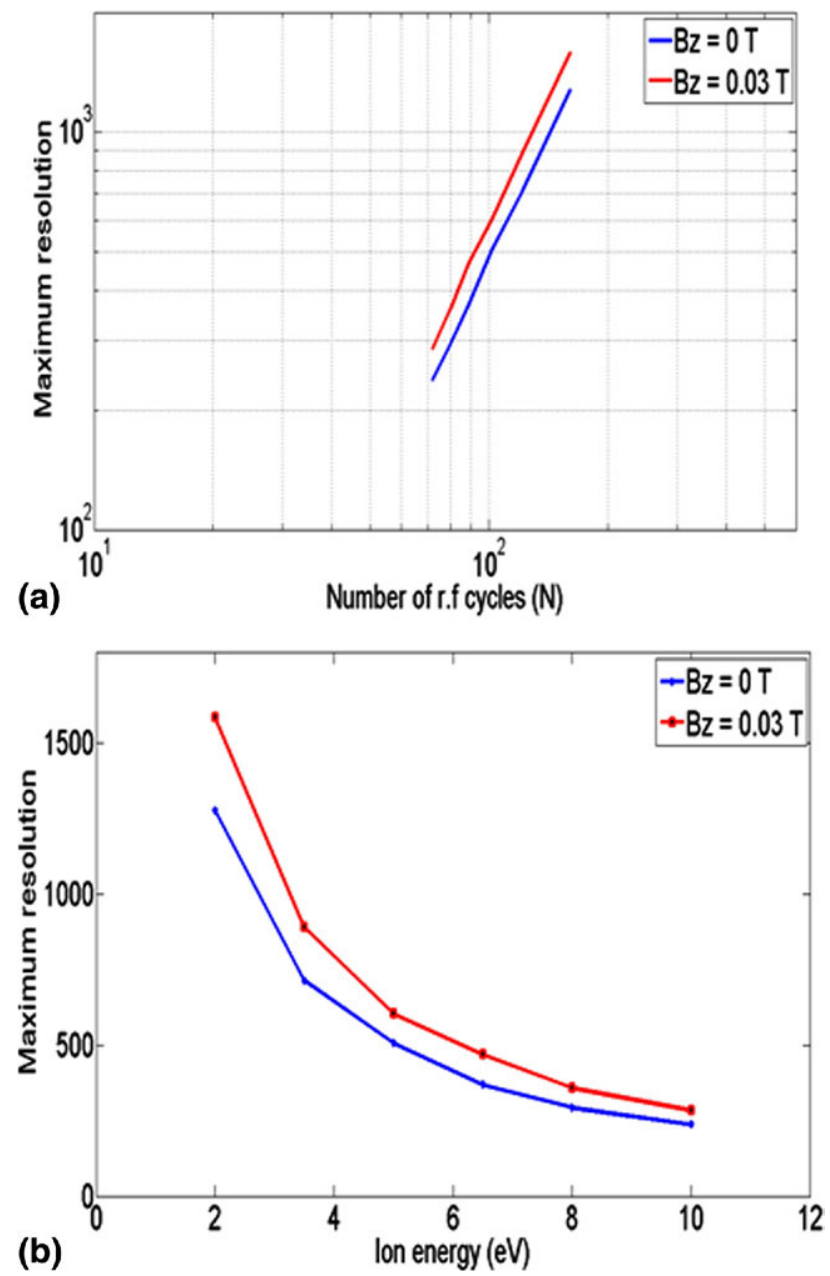

Figure 4. (a) The dependence of maximum resolution on number of rf cycles with and without an applied magnetic field, (b) the dependence of maximum resolution on ion energy with and without an applied magnetic field. 
field the value of $n$ was found to be 2.05 and that of $K$ to be about 27. For simulations with $B_{z}=0.03 \mathrm{~T}$, the value of $n$ was found to be 2.18 and that of $K$ to be about 40 . These results clearly indicate that the predicted performance of the QMS increases in the presence of an axial magnetic field. Figure $4 \mathrm{~b}$ shows the dependence of maximum resolution on ion energy for ${ }^{40} A r^{+}$with and without magnetic field applied. As can be seen from Figure $4 b$, in both cases, resolution decreases with increase in ion energy. However, it can also be observed that percentage increase in resolution with magnetic field decreases as ion energy increases. An increase of $\sim 24.5 \%$ in resolution at 2 $\mathrm{eV}$ can be seen, whereas an increase of only $19.5 \%$ at $10 \mathrm{eV}$ is seen at the same value of magnetic field applied.

The effect can be best demonstrated with the help of ion trajectories in the presence of magnetic field at different ion energies. Figures 5 and 6 show trajectories of ions transmitted with and without magnetic field, at ion energies of 2 and $10 \mathrm{eV}$, respectively. The corresponding traces shown in the figures are for the same ion injected into the QMF at the same point in time (rf phase) and space and at the same point on the mass scale $(\mathrm{m}=40.00 \mathrm{u})$. In both figures the upper trace is for the case with no field applied and lower trace with $B z$ of $0.03 \mathrm{~T}$. In Figure 5 the ion motion originates at $x=$ $-2.03 \times 10^{-4} \mathrm{~m}$ and $y=-2.15 \times 10^{-4} \mathrm{~m}$ with initial velocity of $3.10 \times 10^{3} \mathrm{~m} / \mathrm{s}$. It can be seen from the figure that in the presence of magnetic field at lower ion energy $(2 \mathrm{eV})$ ion motion in both the directions is affected. It is also seen that the amplitude of ion motion in the $x$-direction increases, whereas the amplitude in $y$-direction decreases.

In Figure 6 the ion motion originates at $x=2.94 \times$ $10^{-4} \mathrm{~m}$ and $y=-1.78 \times 10^{-4} \mathrm{~m}$ with initial velocity of $6.93 \times 10^{3} \mathrm{~m} / \mathrm{s}$. It can be observed from the figure that increase in ion energy has a major impact on behavior of ion trajectories in the presence of magnetic field. It can be seen that ion motion in the $x$-direction remains unaffected, while ion motion in the $y$ - direction is modified. It can be concluded that for a QMF with magnetic field applied in the z-direction, the effect of magnetic field on resolution is greater at lower ion energies. This is due to the increased length of time that the ion experiences the magnetic field.

\section{Conclusions}

Experimental results for a low-resolution QMS instrument show an increase in resolution when a static, axial magnetic field is applied along the mass filter in the $z$ direction. This enhanced resolution observed experimentally has been simulated theoretically using our numerical model, which solves the equation of motion for individual ions injected into the QMF with a magnetic field applied. By determining the ion trajectories for a large number of ions $\left(>10^{5}\right)$, mass spectra may be simulated. The effects

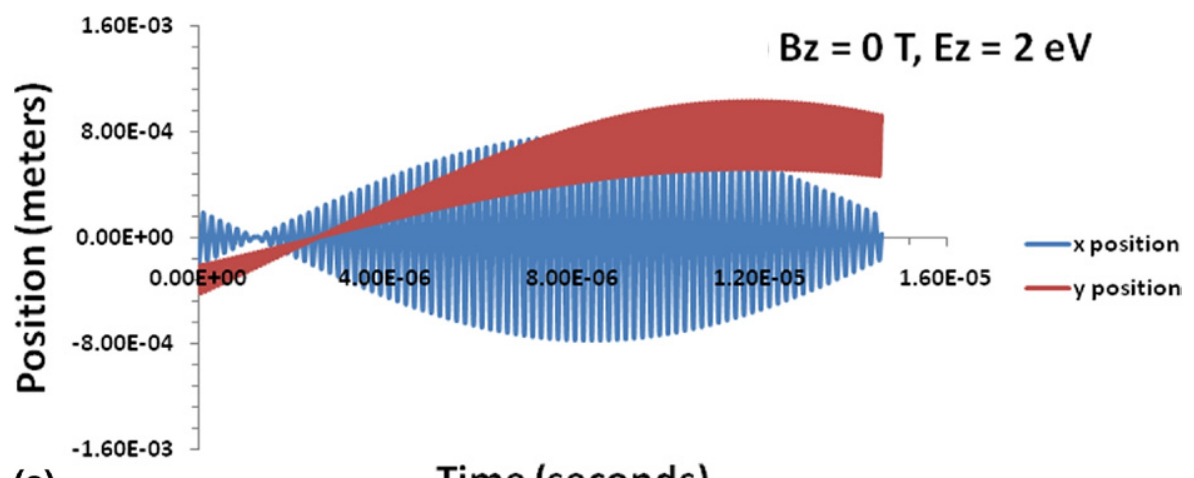

(a)

Time (seconds)

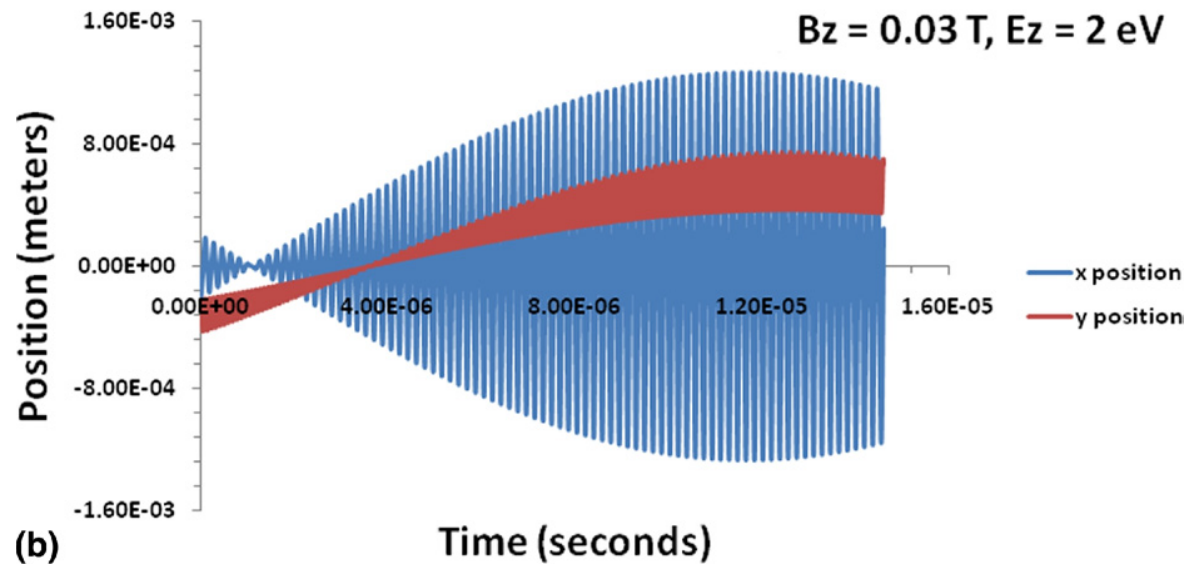

Figure 5. Numerical simulation of ion trajectories in $\mathrm{x}$ and $\mathrm{y}$ directions for ${ }^{40} A r^{+}$with $\mathrm{E}_{\mathrm{z}}=2 \mathrm{eV}$ and with (a) $\mathrm{Bz}=0$ (upper trace) and (b) $\mathrm{Bz}=0.03 \mathrm{~T}$ (lower trace). 


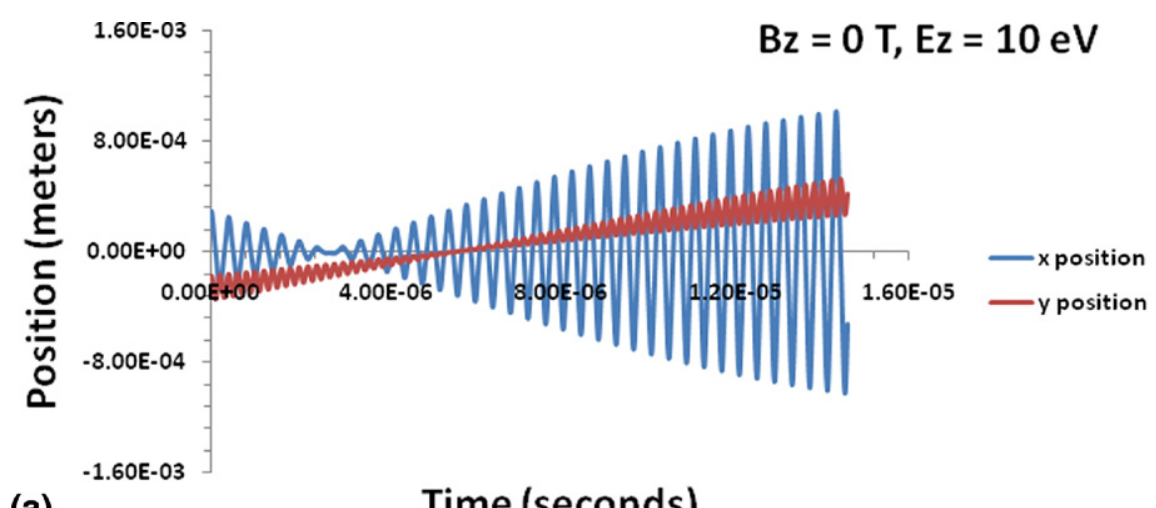

(a)

Time (seconds)

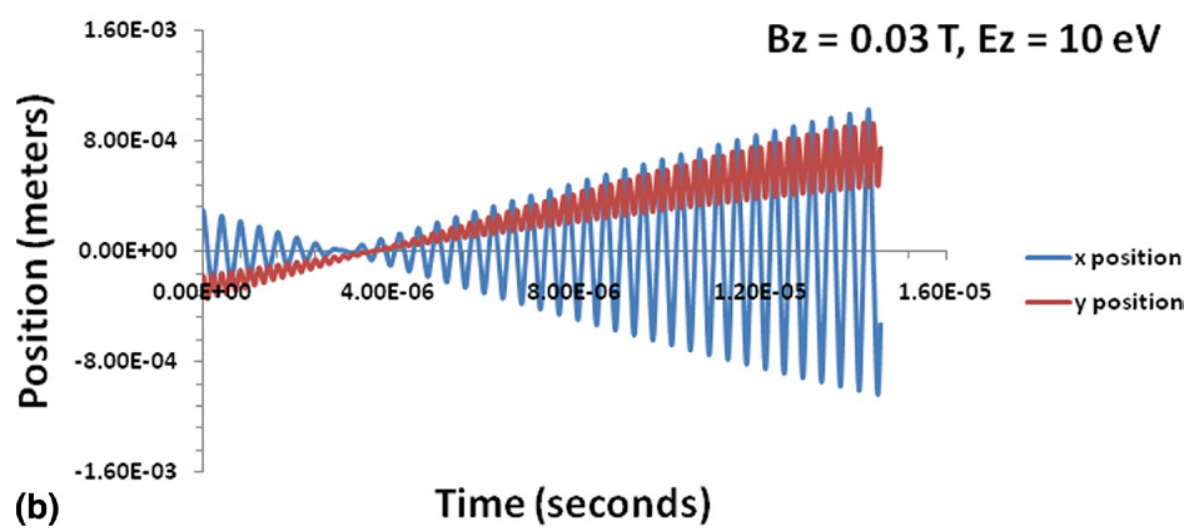

Figure 6. Numerical simulation of ion trajectories in $\mathrm{x}$ and $\mathrm{y}$ directions for ${ }^{40} A r^{+}$with $\mathrm{E}_{\mathrm{z}}=10 \mathrm{eV}$ and with (a) $\mathrm{Bz}=0$ (upper trace) and (b) $\mathrm{Bz}=0.03 \mathrm{~T}$ (lower trace).

may be explained by considering the additional Lorentz force produced by ion motion in the magnetic field. All ions oscillating transversely to the applied magnetic field will experience a Lorentz force in addition to the time varying electrostatic force due to alternating quadrupole field. This results in rejection of ions in the low mass tail as well as high mass tail, giving increased resolution for a given set of operating parameters. High resolutions ( $\mathrm{R}>$ 3000) are predicted for QMFs of length $200 \mathrm{~mm}$ as a result of the applied magnetic field which allows wider instrument application.

\section{References}

1. Blaum, K.; Geppert, C.; Muller, P.; Nortershauser, W.; Otten, E.W.; Schmitt, A.; Trautmann, N.; Wendt, K.; Bushaw, B. A. Properties and Performance of a Quadrupole Mass Filter Used for Resonance Ionization Mass Spectrometry. Int. J. Mass Spectrom. 1998, 181, 67-87.

2. Dawson, P. H. Quadrupole Mass Spectrometry and Its Applications; Elsevier: Amsterdam, 1976; pp 121-152.

3. Douglas, D. J. Linear Quadrupoles in Mass Spectrometry. Mass Spectrom. Rev. 2009, 28, 937-960.

4. Schwartz, J. C.; Senko, M. W.; Syka, J. E. P. A two-Dimensional Quadrupole Ion Trap Mass Spectrometer. J. Am. Soc. Mass Spectrom. 2002, 13, 659-669.

5. Hager, J. W. A New Linear Ion Trap Mass Spectrometer. Rapid Commun. Mass Spectrom. 2002, 16, 512-526.

6. Wiley, W. C.; McLaren, I. H. Time-of-Flight Mass Spectrometer with Improved Resolution. Rev. Sci. Instrum. 1955, 26, 1150-1157.

7. Taylor, S.; Srigengan, B.; Gibson, J. R.; Tindall, D.; Syms, R.; Tate, T.; Ahmad, M. A Miniature Mass Spectrometer for Chemical and Biological Sensing. Proc. SPIE 200, 4036, 187-193.

8. Taylor, S.; Tunstall, J. J.; Leck, J. H.; Tindall, R. F.; Jullien, J. P.; Batey, J.; Syms, R. R. A.; Tate, T.; Ahmad, M. M. Performance Improvements for a Miniature Quadrupole with a Micromachined Mass Filter. Vacuum 1999, 53, 203-206.
9. Dawson, P. H. A Detailed Study of Quadrupole Mass Filter. Int. J. Mass Spectrom. Ion Phys. 1974, 14, 317-337.

10. Batey, J. H. Quadrupole Gas Analyzers. Vacuum 1987, 37, 659-668.

11. Muntean, F. Transmission Study for rf-Only Quadrupoles by Computer Simulations. Int. J. Mass Spectrom. Ion Processes 1995, 151, 197-206.

12. Reuben, A. J.; Smith, G. B.; Moses, P.; Vagov, A.V.; Woods, M. D.; Gordon, D. B.; Munn, R. W. Ion Trajectories in Exactly Determined Quadrupole Fields. Int. J. Mass Spectrom. Ion Processes 1996, 154, 43-59.

13. Ma, F. M.; Taylor, S. Simulation of Ion Trajectories Through the Mass Filter of a Quadrupole Mass Spectrometer. IEE Proc.-Sci. Meas. Technol. 1996, 143(1), 71-76.

14. Voo, A. C. C.; Ng, R.; Tunstall, J. J.; Taylor, S. Transmission Through the Quadrupole Mass Filter: The Effect of Aperture and Harmonics. J. Vac. Sci. Technol. A 1997, 15(4), 2276-2281.

15. Gibson, J. R.; Taylor, S.; Leck, J. H. Detailed Simulation of Mass Spectra for Quadrupole Mass Spectrometer systems. J. Vac. Sci. Technol. A 2000, 18(1), 237-243.

16. Gibson, J. R.; Taylor, S. Prediction of Quadrupole Mass Filter Performance for Hyperbolic and Circular Cross Section Electrodes. Rapid Commun. Mass Spectrom. 2000, 14, 1669-1673.

17. Douglas, D. J.; Konenkov, N. V. Influence of the Sixth and Tenth Harmonics on the Peak Shape of a Quadrupole Mass Filter with Round Rods. Rapid Commun. Mass Spectrom. 2002, 16, 1425-1431.

18. Hogan, T. J.; Taylor, S. Performance Simulation of a Quadrupole Mass Filter Operating in the First and Third Stability Zones. IEEE 2008, 57(3), 498-508.

19. Sreekumar, J.; Hogan, T. J.; Taylor, S.; Turner, P.; Knott, C. A Quadrupole Mass Spectrometer for Resolution of Low Mass Isotopes. J. Am. Soc. Mass Spectrom. 2010, 21, 1364-1370.

20. Tunstall, J. J.; Taylor, S.; Vourdas, A.; Leck, J. H.; Batey, J. Application of Static Magnetic Field to the Mass Filter of a Quadrupole Mass Spectrometer. Vacuum 1999, 53, 211-213.

21. Srigengan, B.; Gibson, J. R.; Taylor, S. Ion Trajectories in Quadrupole Mass Spectrometer with a Static Transverse Magnetic Field Applied to Mass Filter. IEE Proc-Sci. Meas. Technol. 2000, 147(6), 274-278.

22. Gooden, J. K.; Rempel, D. L.; Gross, M. L. Evaluation of Different Combinations of Gated Trapping, rf-Only Mode and Trap Compensation for In-Field MALDI Fourier Transform Mass Spectrometry. J. Am. Soc. Mass Spectrom. 2004, 15, 1109-1115.

23. Kraus, J. D. Electromagnetics; McGraw-Hill: New York, 1991; pp 272-274. 